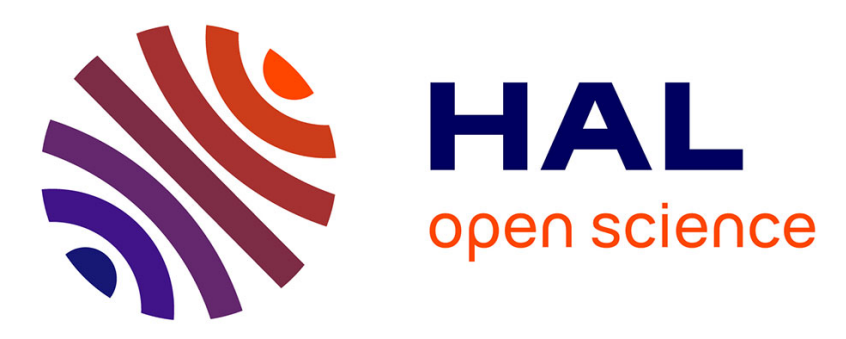

\title{
A study of content based watermarking using an advanced HVS model
}

Florent Autrusseau, Patrick Le Callet, Alexandre Ninassi

\section{To cite this version:}

Florent Autrusseau, Patrick Le Callet, Alexandre Ninassi. A study of content based watermarking using an advanced HVS model. Intelligent Information Hiding and Multimedia Signal Processing, Nov 2007, Kaohsiung, Taiwan. pp.485-489, 10.1109/IIH-MSP.2007.308 · hal-00250662

\section{HAL Id: hal-00250662 \\ https://hal.science/hal-00250662}

Submitted on 12 Jul 2010

HAL is a multi-disciplinary open access archive for the deposit and dissemination of scientific research documents, whether they are published or not. The documents may come from teaching and research institutions in France or abroad, or from public or private research centers.
L'archive ouverte pluridisciplinaire HAL, est destinée au dépôt et à la diffusion de documents scientifiques de niveau recherche, publiés ou non, émanant des établissements d'enseignement et de recherche français ou étrangers, des laboratoires publics ou privés. 


\section{Cover Page}

1) Title of the paper:

\section{A study of content based watermarking using an advanced HVS model}

2) authors' affiliation and address:

IRCCyN-IVC, (UMR CNRS 6597), Polytech' Nantes

Rue Christian Pauc, La Chantrerie, 44306 NANTES, France.

Tel : 02.40.68.30.52

Fax : 02.40.68.32.32

3) e_mail address:

Florent.Autrusseau@univ-nantes.fr

4) Conference \& Publisher information:

IEEE IIH-MSP 2007

http://www.ieee.org/

5) bibtex entry:

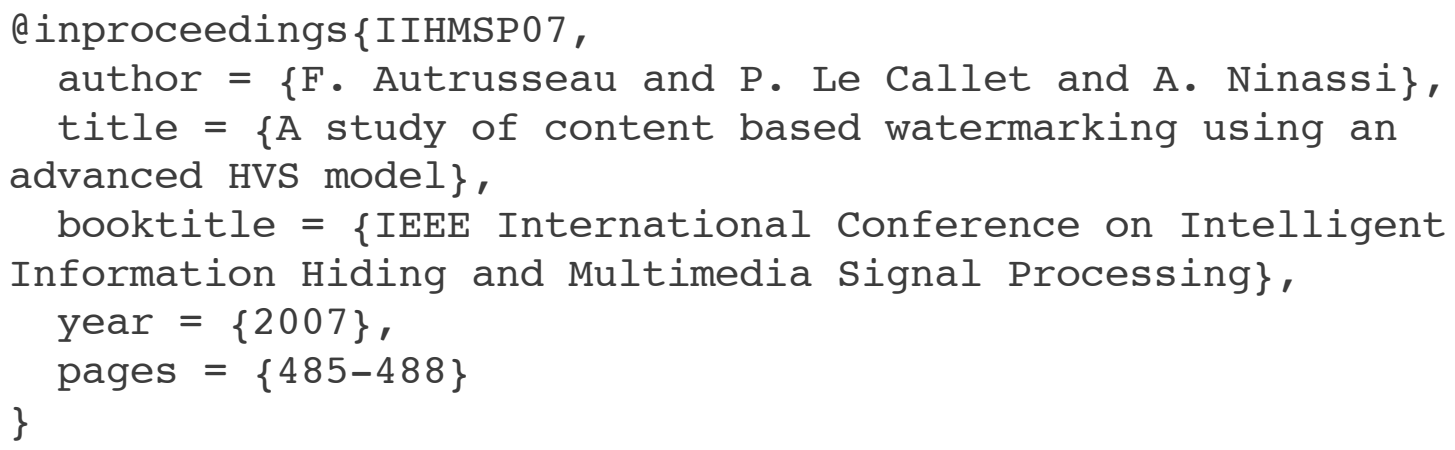




\title{
A study of content based watermarking using an advanced HVS model
}

\author{
Florent Autrusseau, Patrick Le Callet, Alexandre Ninassi \\ IRCCyN Lab., Rue Ch. Pauc, 44306 Nantes, France \\ Florent.Autrusseau@univ-nantes.fr \\ Patrick.Lecallet@univ-nantes.fr \\ Alexandre.Ninassi@thomson.net
}

\begin{abstract}
A key point of image watermarking schemes is to select the image pixels or transformed coefficients where an embedded watermark would reach the optimal invisibility versus robustness trade-off. Human Visual System (HVS) based JND masks are thus useful to optimally adapt the watermark strength right below the visibility threshold. Most of the perceptual masks created so far for watermarking purpose are based on the empirical assumption that edges and/or textures allow the best watermark visual masking and thus ensures good invisibility properties. Nevertheless, although invisibility control in smooth areas is challenging, it could be interesting to extend the watermark in such areas since the robustness performances might increase. The goal of this paper is to use an advanced HVS model to manage invisibility in order to evaluate the robustness of watermarks embedded in smooth areas only, edges/textured areas only or in both areas. This study is further to the one presented in [1]. The robustness was tested against 89 attacked images (using Stirmark), results showed that including smooth areas in the JND mask increases the robustness while preserving good invisibility properties.
\end{abstract}

\section{Introduction}

As previously emphasized by Cox et al. [2], perceptual modeling is very important in watermarking context. It is important to embed the watermark in the perceptually significant image component, otherwise the watermark wouldn't survive several attacks, such as lossy compression algorithms, which strongly modify such components. A few works using perceptual models to improve watermarking schemes have been conducted. Podilchuk and Zeng [3] propose an image adaptive watermarking algorithm for both the DCT and DWT domains. On one hand, the JND masks applied to the DCT domain are issued from quantization matrices established by Watson. On the other hand JND masks used in the DWT domain are computed from visual thresholds given by Watson et al. For both embedding domains, the watermark's robustness have been tested against JPEG compression and cropping. The interest of the watermarking community to Just Noticeable Differences (JND) masks has slowly decreased since a few studies showed that empirical considerations on the human perception could be sufficient for effective watermark embedding [4]. Authors in [5] perform an edges/textures classification, and chose to embed the watermark in such regions, while strictly avoiding smooth regions. Similarly, the author in [6] propose a JND mask design based on the extraction of textures/edges/corners and luminance. The author claim that a more important watermark weighting function may be applied in the textured areas. The JND mask was tested with a mid frequency DCT embedding algorithm. The Noise Visibility Function (NVF) was introduced in [7] and a content adaptive watermarking embedding scheme was designed, such as:

$$
y=x+(1-N V F) \times n \times S+N V F \times n \times S_{1},
$$

where $y$ is the stego image, $x$ is the original one, $n$ stands for a noise-like watermark, $S$ is the watermark strength. Furthermore, the NVF allows an embedding rule in the smooth areas, thanks to the $S_{1}$ variable (which was set to 3 ). The NVF was proved to be very efficient as a weighting function for a noise like watermark. An improved use of the NVF was proposed by the same authors in [8]. Here, the embedding took place in the wavelet domain, which better meets the HVS requirements. In [8], the weighting parameters that are to be applied on either edges/textures or flat image areas ( $S$ and $S_{1}$ in equation 1 ) were experimentally set for each wavelet sub-band. However, unlike previously cited works, the main goal of this paper is to study the ability of the smooth areas to efficiently embed an invisible watermark, that might increase the robustness. A very competitive HVS model is evidently highly desirable in order to successfully embed a watermark in the flat areas of the 
image.

This paper is decomposed as follows: Section 2 briefly explains the watermark embedding technique, that includes a description of the advanced HVS model, section 3 is devoted to the masks creation, and more specifically to the activity classification. And finally, section 4 gives both invisibility and robustness results depending to the embedding areas. Section 5 concludes on the capabilities of the smooth areas to efficiently embed the watermarks.

\section{Mask design and embedding technique}

This work is further to the watermarking algorithm presented in [1]. Basically, the embedding technique (see Figure 1) takes benefit of a frequency decomposition called Decomposition into Perceptual Channels (DPC), which splits the Fourier domain in overlapping sub-bands, modeling the HVS behavior. From a neurobiology point of view, masking effect can be well explained by the receptive fields concept which are tuned to given spatial frequencies and orientation. This can be emulated using a spatial frequency sub band decomposition. Based on psychophysics experiments conducted in our lab, we have derived a Perceptual Channel Decomposition (PCD). The PCD's filters are similar to the cortex filters developed by Watson. However, they have been adapted to a frequency splitting, which is not dyadic according to radial frequencies. Moreover, in this decomposition the angular selectivity is not constant. The PCD uses a set of three band-pass radial frequency channels (crown $I I I$, $I V, V$ ) each being decomposed into angular sectors with an oriented selectivity of $45^{\circ}, 30^{\circ}$ and $30^{\circ}$ respectively. Channel number $I I$ has been merged with the low-pass channel (crown I), which is non-directional, and gives rise to a simple low-pass radial frequency channel (this latter is denoted here as LF). The used Cortex filters are defined as the product between Dom filters which characterize the radial selectivity and Fan filters providing the angular selectivity. The watermark (a square frequency patch) is embedded either in the Fourier domain or in the spatial domain, after being scaled in order to best fit in the visual mask issued from [1]. This leads to the design of a spatial visual mask that define on each site (pixel) the maximum value which can be added without being noticeable.

The local band limited contrast $c_{i, j}(m, n)$ is given by the formula :

$$
c_{i, j}(m, n)=\frac{L_{i, j}(m, n)}{\overline{L_{i, j}}(m, n)}
$$

where $\overline{L_{i, j}}(m, n)$ represents the local mean luminance and $L_{i, j}(m, n)$ the luminance for the $\mathrm{i}, \mathrm{j}$ sub-band and for each $(m, n)$ position (i.e. the spatial representation of all frequencies below the considered sub-band). Based on this

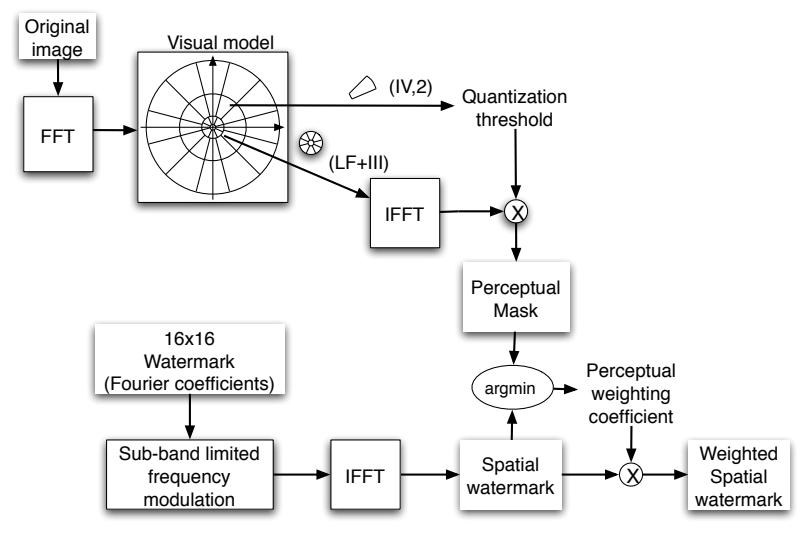

\section{Figure 1. Weighted watermark computation}

contrast definition, the visually optimum quantization of each sub-band content can be performed with a uniform law. Optimum quantization step $\Delta C_{i, j}$ has been identified from psychophysics experiments for each $(i, j)$ subband based visual thresholds. Interested readers may refer to previous works for further details on the mask creation [1]. From these observations, we can define visual mask for each $(i, j)$ sub-band providing the maximum luminance variation $\Delta L_{i, j}(m, n)$ that can be applied to each $(m, n)$ site without introducing visible artifacts. $\Delta L_{i, j}(m, n)$ is then given by

$$
\Delta L_{i, j}(m, n)=\Delta C_{i, j} \times \overline{L_{i, j}}(m, n)
$$

The watermark amplitude must be weighted according to the visual mask. Although the visual mask is spatially defined, the Fourier transform linearity allows to use the same weighting coefficient independently in spatial or Fourier domain. A Fourier coefficients watermark is then built and modulated onto a frequency carrier. Finally a perceptual weighting coefficient $K_{i, j}$ is computed from the watermark's spatial domain representation and the sub-bands dependent visual mask. $K_{i, j}$ is given in equation 4

$$
K_{i, j}=\operatorname{argmin}_{m, n}\left(\left|\frac{\Delta L_{i, j}(m, n)}{W_{S}(m, n)}\right|\right)
$$

where $\Delta L_{i, j}(m, n)$ represents the previously defined visual mask and $W_{S}(m, n)$ depicts the watermark's spatial representation before weighting process by factor $K_{i, j}$ for each $(m, n)$ spatial position. It is very important to notice that the JND masks proposed by this technique are suitable for specific frequency contents. This means that for a chosen sub-band, the frequency content of the embedded watermark should ideally be totally restrained in the same frequency content. Figure 1 summarizes both the perceptual mask creation steps (upper branch) and the watermark weighting process (lower branch). The detection pro- 
cess performs a cross-correlation between the stored watermark and the Fourier coefficients surrounding the known frequency carrier extracted from the marked image. This cross-correlation values are then compared to a detection threshold in order to certify the watermark presence in the modified coefficients. The only needed data for the retrieval procedure are the original watermark, and its frequency carrier.

\section{Adaptation of the technique based on cate- gory selection}

Once the JND mask values obtained for every pixel of the input image (equation 3), two binary masks were created depending on the image activity. These masks will be denoted as $M_{S}$ and $M_{T}$ in the following, and they will respectively represent the smooth and activity areas. As the main goal of this work is to study the watermarking performances depending on the image activity, an ad hoc supervised activity classification was performed.Basically, the image was classified by thresholding the variance in a slided window with different sizes combined with orientation detectors. The so-obtained classification is not exhaustive, e.g. some pixels may not be assigned to any of the previously mentioned categories. The two binary masks $M_{S}$ and $M_{T}$

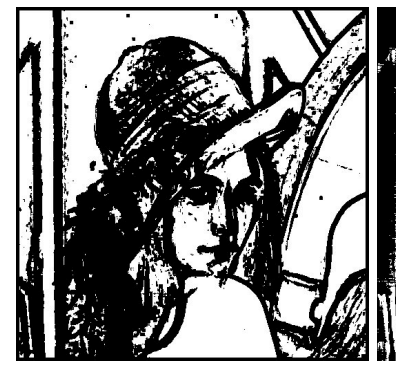

(a)

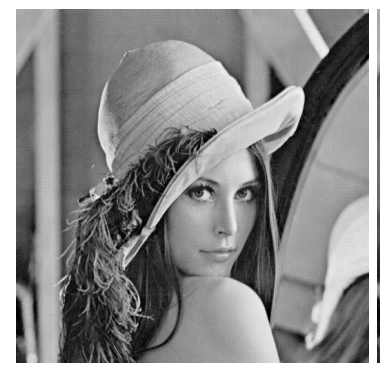

(c)

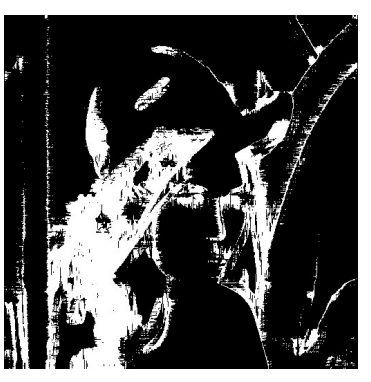

(b)

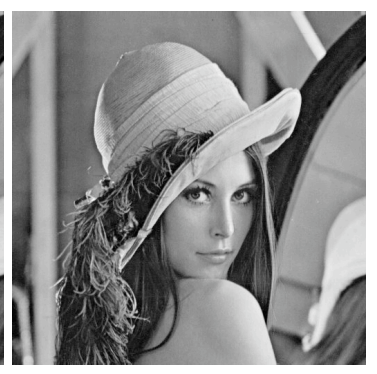

(d)
Figure 2. The two computed binary masks, i.e smooth areas, $M_{S}(\mathbf{a})$, and textures $M_{T}$ (b) along with the corresponding marked images (c) and (d). are depicted on figure 2. The watermark is weighted according to the JND mask, and multiplied by the binary masks prior to the embedding process. This leads to two independently watermarked images (provided in figure 2(c) and (d)), the detection process is then applied on each of these images.

\section{Results}

The watermark detection is performed in the Fourier domain, computing the cross-correlation functions between the patch surrounding the supposed watermark frequency carrier of the marked image, and the stored watermark patch. Considering $16 \times 16$ frequency watermarks, previous experiments [1] demonstrated the best detection for a threshold set to 0.25 . We thus chose this threshold value in the following. Once the detection threshold is set, the cross-correlation is computed for every attacked image and for each activity based watermarking. Figure 3 presents for all 89 Stirmark attacks [9], the detection performances. For each tested image, the cross-correlation was computed between the original watermark and the portion of the image's spectrum presumably containing the mark. The Y-axis represents the obtained cross-correlation coefficients, whereas the $\mathrm{X}$-axis represents all the attacks. For a better understanding of the indexes in the $\mathrm{X}$-axis, please, refer to the works in [1]. Three test images were used ("lena", "boats" and "plane"). Figure 3 presents the robustness results depending on the chosen activity areas for images "lena" (Fig. 3(a)) and "boats" (Fig. 3(b)). Robustness was also computed in the full JND mask, no activity based segmentation is performed (refers directly to the works in [1]). According to the previously determined detection threshold, Figure 4 presents the number of correctly detected watermarks over the full set of attacks and depending on the image activity. The smooth areas embedding appears to provide good detection capabilities.

Weighted PSNR (wPSNR) was used to assess the perceived quality of the marked images. Results are given in figure 5 for each watermarked image. Figure 5 shows, as expected, best invisibility results in the textured areas, it is however important to notice that good wPSNR measures are also obtained in the smooth areas.

\section{Conclusion}

This work was further to [1]. The goal was here to evaluate the invisibility versus robustness trade-off according to the image activity. The embedding technique takes into account an advanced perceptual model, embedding was respectively applied to smooth image areas or textures. For 


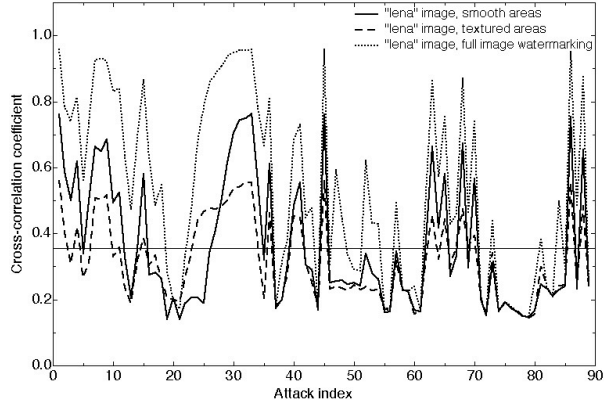

(a)

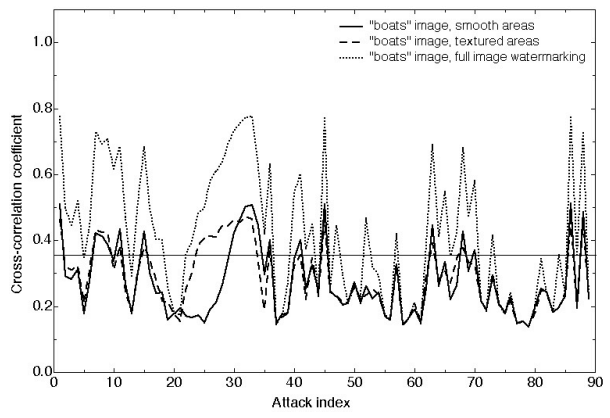

(b)

Figure 3. Robustness for two input images against all Stirmark attacks $(16 \times 16$ watermarks are used).

each of the tested areas, detection results are given for all Stirmark attacks, and quality assessment is performed. Preliminary results showed that a JND mask including smooth areas may increase the robustness capabilities, without introducing visible artifacts.

\section{References}

[1] F. Autrusseau and P. Le Callet, A robust image watermarking technique based on quantization noise visibility thresholds, Signal Processing, 87(6), June 2007, pp. 1363-1383.

[2] I. J. Cox, M. L. Miller, A review of watermarking and the importance of perceptual modeling, Proc. SPIE Conf. on Human Vision and Electronic Imaging II, 3016 (1997) 92-99.

[3] C. I. Podilchuk, W. Zeng, Image-adaptive watermarking using visual models, IEEE Journal on Selected Areas in Communications, 16 (4), (1998) 525-539.

[4] F. Bartolini, M. Barni, V. Cappellini, A. Piva, Mask building for perceptual hiding frequency embedded wa-

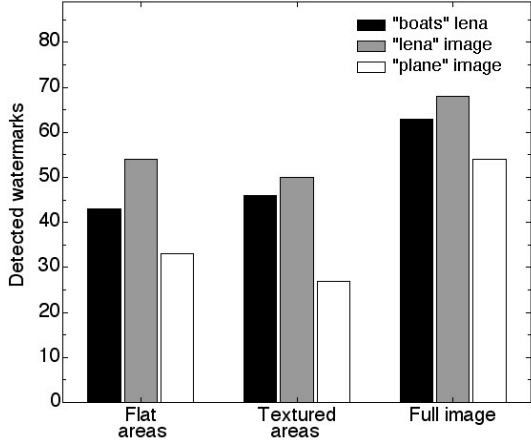
Figure 4. Detection results for the three
tested images

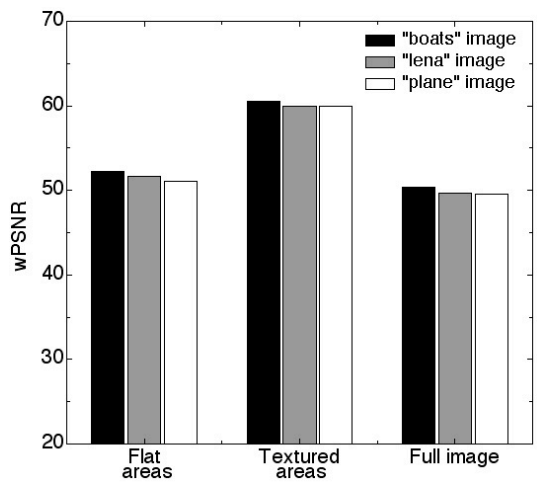

Figure 5. wPSNR plotted according to the chosen embedding areas

termarks. International Conference on image Processing, 1, pp. 450-454, 1998.

[5] M. S. Kankanhalli, Rajmohan, K. R. Ramakrishnan. Content based watermarking of images. ACM Multimedia - Electronic Proceedings, 1998.

[6] A. Parthasarathy, Improved Content based image watermarking, ArXiv Computer Science e-prints, 2006.

[7] S. Voloshynovskiy, A. Herrigel, N. Baumgaertner and T. Pun, "A Stochastic approach to content adaptive digital image watermarking", Intl. Workshop on Info Hiding, Vol. LNCS 1768, pp. 212-236, Springer Verlag, 29 September -1 October 1999.

[8] S. Voloshynovskiy, F. Deguillaume and T. Pun, "Content adaptive watermarking based on a stochastic multiresolution image modeling", European Signal Processing Conference (EUSIPCO'2000), Sep 5-8, 2000.

[9] F. A.P. Petitcolas, R. J. Anderson, M. G. Kuhn, Attacks on Copyright Marking Systems, Second Workshop on information hiding, 1525 (1998) 218-238. 